\title{
Automatic Test Generation on a (U)SIM Smart Card
}

\author{
Céline Bigot $^{1}$, Alain Faivre ${ }^{2}$, Christophe Gaston ${ }^{2}$, and Julien Simon ${ }^{1}$ \\ ${ }^{1}$ Oberthur Card Systems, R\&D - EMC, 71-73 rue des Hautes-Pâtures, \\ 92726 Nanterre Cedex, France \\ \{c.bigot, j.simon\} @oberthurcs.com \\ ${ }^{2}$ CEA/LIST, Saclay, F-91191 Gif sur Yvette Cedex, France \\ \{alain.faivre, christophe.gaston\}@eea.fr
}

\begin{abstract}
Usually, testing smart card software is carried-out by specialized engineers in a proprietary language. Testing represents generally half of smart card development effort. With the increasing use of semi-formal and formal modeling languages, such as UML, and the emergence of automatic test generators in the industry, we have studied a way to adapt these techniques for smart card. In this article, we present an automatic test generator, named AGATHA, and its architecture, which can handle UML specifications. Then, we suggest a way to model (U)SIM smart card functionalities in UML. We use the test generator on our (U)SIM smart card UML models and automatically produce our first test cases.
\end{abstract}

\section{Introduction}

It's not necessary to remind that in any industry, the later a bug is discovered in a development process, the more it costs to correct it. Today, in the smart card industry, half of the effort of the development activity is devoted to testing. Testing includes:

- unit testing, carried out during the programming activity by programmers, which ensures that each elementary item has a correct behaviour and rules out basic programming errors,

- $\alpha$-testing, carried out after the programming activity by $\alpha$-testers, which ensures that smart cards have a correct behaviour compared with the functionalities described in the specifications,

- $\beta$-testing, carried out after $\alpha$-tests by $\beta$-testers, which ensures that smart cards in mobile phones, in payment machines or in any other devices also comply with the specifications.

In the context of this long and complex process, handwritten by programmers and testers, we would like to study the possible automatic generation of a part of these tests. Our first idea, described in this article, consists in taking into account the $\alpha$-test activity. By automatic test generation, we expect to increase the coverage and the quality of the tests in order to ensure a complete validation of the specification.

Moreover, with the increase of system complexity, it's difficult between two versions of a project to know which tests evolve, which ones are obsolete, etc. It's also difficult for a non-tester to understand produced tests. Thus, our idea is to combine automatic test generation with a simple formalism to represent test specifications and their evolution. 
Methods and tools required for validation are not recent, and a lot of researches has been done to try to fill the deficiency. For test generation, we can take as examples [11], [19], [2] and [32]. Semi-formal and formal methods, such as UML [30], B [1] or SDL [20], allow an abstract design for a behavioural specification of the system under test. Thanks to simple, expressive and abstract notations, textual or graphical, we can easily use these types of formalisms to design smart cards. Moreover, these formalisms allow the use of existing validation tools.

The last few years, several studies were conducted on design and validation of smart card software. For example, [8] represents results on the CEPS standard, [3] shows validation results on the GSM 11.11 standard [12], [31] used automated test generation on the WAP Identity Module, [5] describes techniques which can be apply at different levels of smart card software, [6] represents an automatic test generation with the LEIRIOS tool [26] from B specifications, [29] presents a method to automatically generate test for Java card applets and [7] offers a semi formal model of Java Card applications in UML.

In our context, we would like to use a more simple and graphical formalism, which can be used by any engineer. With the emergence of UML in industry and the multiple types of diagrams offered, this formalism represents a good alternative. In the panel of automatic test generators (see [33] for examples of automatic test generators), we were interested by the symbolic approach of the AGATHA $^{1}$ tool [17], [25], [34], [4], [28] and [14], developed at the CEA ${ }^{2}$-List.

Therefore, the article is organized as follows. First, we present the AGATHA tool and the automatic test generation. Second, after presenting how we can model a part of smart card in UML, we describe the use of AGATHA on our semi-formal models and present our first results on a PIN command. We finally conclude and explain our future actions.

\section{AGATHA, an Automatic Test Generator}

There exists several ways to validate system specifications. A first one consists in theorem proving and model checking [9]. These kinds of techniques have successfully proved their use for the validation of critical systems. But two major drawbacks of these techniques remain: for model checking, the combinatorial explosion due to variable domains, and, for theorem proving, the need of high-level skills from the developer, who must be aware of formal method foundations.

Automatic test generation is another way to tackle the problem of system validation. Compliance testing is the most well known part of this domain, which consists in verifying that a system matches its specification. Our first purpose is to validate a system specification, and generate tests in order to execute them on the specification and possibly on the system itself.

Most validation tools use enumerative techniques and are therefore limited by the combinatorial explosion problem when trying to exhaustively identify the numerical

1 AGATHA : "Atelier de Génération Automatique de Test Holistiques à partir d'Automates" Automatic holistic tests generation framework for automates.

2 CEA: "Commissariat à l'Energie Atomique" - French atomic energy reseach center. 
behaviours of a system. Several validation tools focus on verification on particular aspects: test purpose [15], temporal properties [36], etc.

The solution proposed by AGATHA is exhaustive symbolic path coverage. Test generation allows detecting specification deadlocks, unreachable transitions, losses of messages, etc. Moreover, the AGATHA toolset is designed to be as transparent as possible in order to reduce the effort of detection and comprehension of errors. In that context, it is not necessary to be an expert in formal methods, as for model checkers or theorem provers, to interpret AGATHA results and to correct specifications or implementations.

The following subsections present the AGATHA architecture and an overview of the different academic techniques used in order to reach minimal exhaustive path coverage.

\subsection{General Principles}

The AGATHA approach intends to help conception and validation of formal specifications modelled with communicant automata systems. Thus, with symbolic execution techniques, AGATHA computes the exhaustive symbolic behaviour graph of the specification. Then, from this graph, it generates test cases used to debug the specification or to validate the implementation, along with an incremental conception process.

Figure 1 details the AGATHA general architecture.

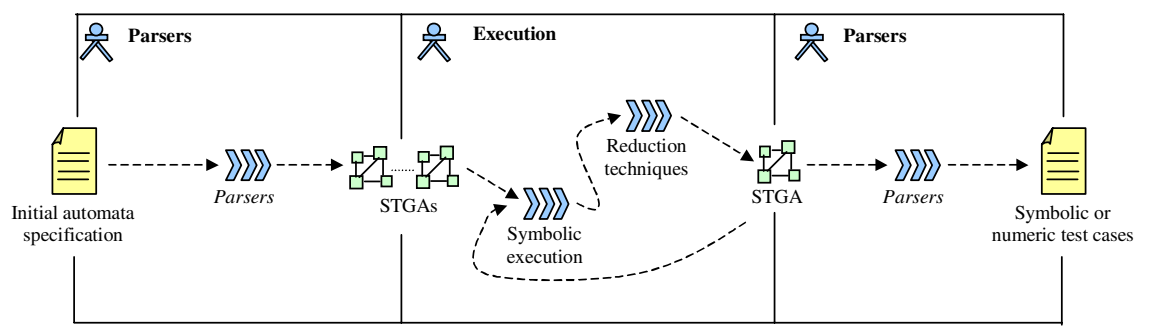

Fig. 1. AGATHA general architecture

The tool treats automata specifications and translates it into its internal language, called STGA (Symbolic Transition Graph with Assignment) [27]. This translation allows the symbolic execution of the specification as defined in [18]. Thus, it allows obtaining an exhaustive behaviour graph of the specification. Thanks to reduction techniques defined in [25] and in [34], with the help of the rewriting tool Brute [21], the graph is reduced in a particular STGA. On this particular STGA, AGATHA uses a constraint solver Omega [23] providing for each path of the graph corresponding to a symbolic behaviour, one or more numerical test cases.

\subsection{Main Principle: Symbolic Execution}

At the beginning, symbolic execution has been proposed in [24] and in [10] to construct structural tests for sequential programs. The main idea of symbolic execution 
consists to use symbols as entry data of the program, denoting any entry data, instead of numerical values and to interpret the entry language in a way that allows manipulating symbolic expressions instead of numerical ones. AGATHA uses an adaptation of symbolic execution to generate tests from specifications based on automata.

The input language of AGATHA is based on the STGA formalism [27], which is a symbolic transition graph. Like graph formalisms, a STGA includes states and transitions. This type of graph allows representing in an abstract manner all behaviours of a specification. Transitions represent events that allow the evolution of the system: events can be received or emitted. Triggering a transition can be conditioned by a logical expression and system variables can evolve.

STGA Example. Figure 2 presents a STGA example of an elevator system. It contains four states and seven transitions. The initial state is $\mathrm{Q}_{0}$.

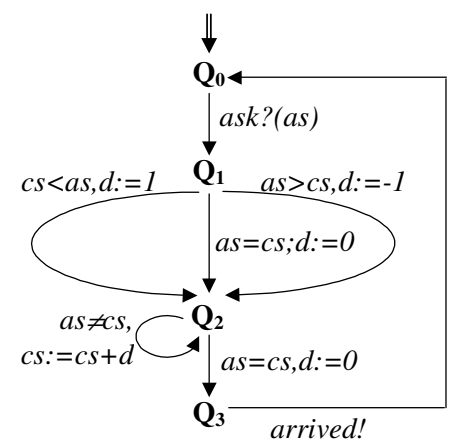

Fig. 2. STGA sample of an elevator system

To trigger the output transition of $\mathrm{Q}_{0}$, the elevator system awaits the reception of the message ask, denoted ask?(as), which represents a call to a stage by the user in the cabin which is stored in the as variable. After the triggering of the transition, the system is in the state $Q_{1}$. $Q_{1}$ has three output transitions towards $Q_{2}$. The left one is conditioned by the logical expression: $c s<a s$ meaning the asked stage $a s$ is over (< sign) the current stage represented by the $c s$ variable. The right one is conditioned by: cs $>$ as meaning the asked stage is under the current stage. The middle one is conditioned by: $c s=a s$ meaning the asked stage is equal to the current one. In the first case, the elevator moves up the cabin, which is materialized by the operation $d:=1$ ( $\mathrm{d}$ represents the direction), while in the second case, the elevator moves down the cabin, which is materialized by $d:=-1$. In the third one, the elevator leaves the cabin at its current stage, which is materialized by $d:=0 . \mathrm{Q}_{2}$ has two outgoing transitions: one with $\mathrm{Q}_{2}$ for target and one with $\mathrm{Q}_{3}$. The transition with $\mathrm{Q}_{2}$ for target is conditioned by the logical expression: $a s \neq c s$ and increases the current stage $c s$ with the direction $d$, materialized by the operation $c s:=c s+d$. This transition means that as long as the current stage is different from the asked stage, the cabin has to continue to move up or down. The second transition is conditioned by: $a s=c s$ and initializes the direction $d$ to 0 . This transition means that if the current stage is equal to the asked stage, the cabin 
is stopped. The $\mathrm{Q}_{3}$ outgoing transition allows to come back to $\mathrm{Q}_{0}$ and tells the user in the cabin that the elevator has reached the asked stage, which is represented by the emission of the message arrived, denoted arrived!.

In the AGATHA context, [25] redefines symbolic execution for STGA using the approach defined in [19]. Thus, symbolic execution simulates the behaviour of a STGA specification in assigning symbolic values to variables instead of numerical ones. Then, the specification is executed according to the semantics of each instruction and communication.

The general principle of symbolic execution consists in computing symbolic states of a system, each of them being denoted by a couple (guard, symbolic memory), where:

- guard is the condition needed to reach this symbolic state,

- symbolic memory is a function which associates to each variable of the system an expression based on symbolic input values.

The expression associated to a variable in a symbolic state corresponding to an execution path, from the initial state of the system, is computed by interpreting one by one instructions met all along this execution path. The associated guard is composed of the conjunction of all the execution conditions (denoted by constraints on symbolic input values) met all along the considered execution path. This guard is called a path condition or PC. To simplify this type of expression, AGATHA uses the simplifier Brute [21] extracted from CafeOBJ tool of $\mathrm{JAIST}^{3}$. This is a rewriting tool, which transforms terms in normal forms with the help of a set of rewriting rules and evaluation strategies defined by the AGATHA user.

The result of a symbolic execution is a symbolic execution tree where each path represents the symbolic evolution of all variables according to initial symbolic values. Each path is a particular behaviour of the STGA specification.

Symbolic Execution Example. In our example of the elevator system presented in Figure 2, an extract of the symbolic execution tree computed by the AGATHA symbolic execution is presented in Figure 3.

At the initialization, the STGA specification obtained by symbolic execution is in a state corresponding to the initial state $\mathrm{Q}_{0}$ of the elevator system. The elevator specification manipulates the variables: $a s, c s$ and $d$ on which there are no initial constraints. A symbolic constant is assigned to each variable: $\mathrm{a}_{0}, \mathrm{~b}_{0}$ and $\mathrm{c}_{0}$ (resp.) on which there are no initial constraints, denoted by the condition true. The elevator system can evolve if it receives the ask message with a value. This value is stored in the variable as and is supposed to have the symbolic value $\mathrm{a}_{1} . \mathrm{a}_{1}$ is assigned to the as variable. As the trigger of the transition is not conditioned, the condition to reach this second symbolic state is always true (true $\wedge$ true $=$ true).

To leave the state $\mathrm{Q}_{1}$, there are three transitions. So to leave the symbolic state $\mathrm{Q}_{1}$, there are also three transitions.

As the first transition is conditioned by the expression: $c s<a s$, and, as $c s=b_{0}$ and $a s=a_{1}$ in this state, the condition to reach the symbolic state $\mathrm{Q}_{2}$ corresponds to $b_{0}<a_{1}$. On this transition, 1 is also assigned to $d$, which is reflected in the symbolic state.

3 JAIST: Japan Advanced Institute Technology. 


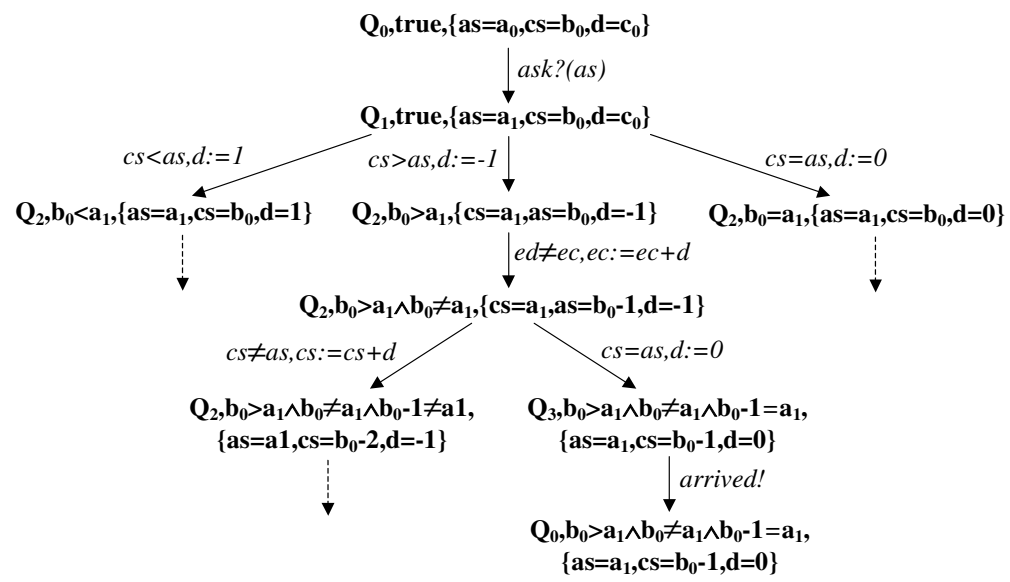

Fig. 3. Extract of the symbolic execution of the elevator system

As the second transition is conditioned by the expression: $c s>a s$, the condition to reach the symbolic state $\mathrm{Q}_{2}$ corresponds to $b_{0}>a_{1}$ and -1 is assigned to $d$.

As the third transition is conditioned by the expression: $c s=a s$, the condition to reach the symbolic state $\mathrm{Q}_{2}$ corresponds to $b_{0}=a_{1}$ and 0 is assigned to $d$.

To leave the symbolic state $\mathrm{Q}_{2}$ where $\mathrm{d}=-1$, two transitions have to be considered. The first one is conditioned by: $a s=c s$ and the second one by: $a s \neq c s$. However, as $b_{0}>a_{1}$, predictably as $\neq c s$ and only the second transition can be triggered. The output transition of the symbolic state $\mathrm{Q}_{2}$ where $\mathrm{d}=-1$ leads to another symbolic state $\mathrm{Q}_{2}$ reached if the condition: $b_{0}>a l$ and $a s \neq c s$ is verified and such as $a s=a_{1}, c s=b_{0^{-}} l$ and $d=-1$. In this state, we can also trigger the two same transitions. As the symbolic value of $c s$ evolves, the two transitions can be triggered. The first one leads to the symbolic state $\mathrm{Q}_{3}$ and the second one to another symbolic state $\mathrm{Q}_{2}$. The trigger of the first transition implies that the condition to verify to reach the symbolic state $\mathrm{Q}_{3}$ is: $b_{0}>a l$ and as $\neq c s$ and $a_{1}=b_{0^{-}}-1$ and $a s=a_{1}, c s=b_{0^{-}} 1$ and $d=0$. To leave this state the only transition is conditioned by the emission of the message arrived and allows the system to come back to state $\mathrm{Q}_{1}$.

The other steps of the computation are based on the same principle.

\subsection{Further Techniques}

As the symbolic execution tree represents all behaviours of a specification, its construction is subordinated to reduction procedures in order to eliminate as many redundant paths as possible. There exists different tactics such as:

- use a classical graph coverage, as for example a transition coverage (the symbolic execution stops when all the transitions are triggered once if possible), a state coverage, a path coverage, etc,

- cut "empty" path conditions when detected both from a Boolean criteria or polyhedral criteria. AGATHA uses the Omega constraint solver, based on Presburger theory [23] to achieve that, 
- avoid computation of a path deductible from another modulo an interleaving detection less sophisticated than in [35]: an internal transition without any temporal constraint with other transitions,

- compute comparison procedures between symbolic nodes and, if necessary for the current calculated nodes, refer to an already existing symbolic node.

These procedures are necessary to avoid the state explosion problem.

AGATHA uses several heuristics to compute comparison procedures for each symbolic node:

- an equality procedure: two symbolic nodes are considered as equivalent if the corresponding control nodes are the same and the symbolic guards are syntactically equal,

- an inclusion procedure: two symbolic nodes are considered as equivalent if the corresponding control nodes are the same and if the polyhedron induced by variable domains defined by the guard of one is included in the other polyhedron,

- an equivalence procedure: two symbolic nodes are considered as equivalent if the corresponding control nodes are the same and if polyhedrons induced by variable domains defined by guards are equal.

As symbolic expressions of variables may also quickly grow, a last simplification procedure must be applied "on-the-fly" in order to shorten expression and to detect useless paths [16]. We use the simplifier Brute, based on rewriting techniques. These rewriting rules actually composed of more than three hundred rules, allow both to maintain symbolic expressions within a reasonable size range and to obtain normal forms of expressions, easing the comparison between expressions needed by algorithms such as comparison procedures.

Other tactics and reduction techniques have been introduced in [34] and in [33]. Generally, a mix of the different tactics is used to obtain the minimal result required to guarantee the entire coverage of the specification.

\subsection{Test Extraction}

Symbolic test cases are extracted from the symbolic execution tree. As each path of the tree represents a symbolic behaviour of the specification, a test case is extracted from each leaf of this symbolic tree. From each symbolic test case, one or more numerical test cases may be produced with the help of constraint resolution techniques used on the path condition associated to the tree leaves. The constraint solver is used to extract the symbolic value of each variable with the associated path condition and to generate numerical values, which respect the path condition. The choice of the constraint solver connected to AGATHA depends on the applicative context. For example, we can use the Omega tool [23].

Test Extraction Example. With the symbolic execution of the elevator system, Figure 3, we identify the path: $\mathrm{Q}_{0} \mathrm{Q}_{1} \mathrm{Q}_{2} \mathrm{Q}_{2} \mathrm{Q}_{3} \mathrm{Q}_{0}$, which represents the symbolic test case such as the asked stage, is under the current stage of one stage. To generate a numerical test case corresponding to this symbolic test case, we have to find numerical values for: $a_{0}, a_{1}, b_{0}$ and $c_{0}$ which verify the path condition: $b_{0}>a_{1} \wedge b_{0} \neq a_{1} \wedge b_{0}-1=a_{1}$. 
For example, we can choose: $\mathrm{a}_{0}=2, \mathrm{a}_{1}=3, \mathrm{~b}_{0}=2$ and $\mathrm{c}_{0}=0$.

Any other series of numerical values verifying the path condition is valid and forms a possible numerical test case. Techniques used by AGATHA allow considering that every numerical test case contained in a symbolic one are equivalent. So, only one numerical test case by each symbolic one is required to cover all the specification.

Moreover, note that the size of our elevator, which is not defined, doesn't step in the symbolic computation. Thus, our specification allows representing an elevator with two, three or more stages.

These test cases can be simulated either on industrial tools that allow generating specifications or on implementations. It often requires an adjustment to the adequate formats.

\section{Application to (U)SIM Smart Card}

The aim of this article is to study the utility of the AGATHA tool in the smart card environment. To begin our experience, we shall limit our domain to (U)SIM smart cards. For (U)SIM smart cards, there are different standards, which describe a lot of card features. Function specifications are described in the 3GPP 11.11 standard [12] and tests on these functions are described in the 3GPP 11.17 standard [13]. For Oberthur Card Systems, a test case is a sequence of instructions in a proprietary language, using hexadecimal codes.

In this section, we propose a UML representation for test cases of (U)SIM smart card behaviours. Then, we present results obtained by the application of AGATHA on these UML models.

\subsection{A UML Representation for (U)SIM Smart Card Tests}

As smart card tests consist in sequences of instructions and as we would like to represent smart card tests and smart card behaviours, we propose to use UML state diagrams. For the moment, we only use this type of diagram. It is very intuitive and can be learned very quickly even by a UML uninitiated: it's a sort of automata language, with states and transitions. The trigger of a transition can be conditioned by a message reception, a message emission, a logical expression, etc. For our work, we only use a sub-part and not the entire power of state diagram notations.

Suppose that we would like to represent a test case from the 3GPP 11.17 standard. As for a function, the test is a sequence of instructions. Our corresponding UML state diagram reflects this sequence. In some instances, we can identify sub-parts in a test case and represent these sub-parts in the UML state diagram, as described in the following example.

The CHANGE CHV Function Example: UML representation for test cases. Above all, we recall the CHANGE CHV specification extracted of the 3GPP 11.11 standard ([12] p.34):

4 chv: Card holder verification information; access condition used by the SIM for the verification of the identity of the user. 
The CHANGE CHV function assigns a new value to the relevant $\mathrm{CHV}$ subject to the following conditions being fulfilled: $\mathrm{CHV}$ is not disabled; $\mathrm{CHV}$ is not blocked.

The old and new $\mathrm{CHV}$ will be presented.

1) If the old $\mathrm{CHV}$ presented is correct, the number of remaining $\mathrm{CHV}$ attempts for that $\mathrm{CHV}$ will be reset to its initial value 3 and the new value for the $\mathrm{CHV}$ becomes valid.

2) If the old $\mathrm{CHV}$ presented is false, the number of remaining $\mathrm{CHV}$ attempts for that $\mathrm{CHV}$ will be decremented and the value of the $\mathrm{CHV}$ remains unchanged. After 3 consecutive false CHV presentations, not necessarily in the same card session, the respective $\mathrm{CHV}$ is blocked and the access condition can never be fulfilled until the UNBLOCK CHV function has been performed successfully on the respective $\mathrm{CHV}$.

Input: indication CHV1, old CHV1, new CHV1.

Output: none.

The test case of the CHANGE CHV function, extracted from the 3GPP 11.17 standard (see [13] pp.65-67), is composed of:

1) An incorrect $\mathrm{CHANGE} \mathrm{CHV}$, steady of a status verification: how much attempts remained, a correct $\mathrm{CHANGE} \mathrm{CHV}$ and a status verification,

2) Two incorrect CHANGE CHV, steady of a reset, an incorrect CHANGE $\mathrm{CHV}$, a reset, an incorrect $\mathrm{CHANGE} \mathrm{CHV}$ and a correct UNBLOCK CHV,

3) A correct DISABLE CHV, an incorrect CHANGE CHV and a correct ENABLE CHV.

A correct function is characterized by a returned status 9000 and an incorrect one is characterized by a returned status 9804 or 98 40, this one meaning that the smart card is blocked. Status verification is done by comparison of expected data and effective data.

The corresponding state diagram is presented in Figure 4 . The initial state is $\bullet$. We suppose that the output transition from the initial state to state A contains data for initializing smart card such as the personalization. A holds two output transitions.

The right one to $\mathrm{C}$ is conditioned by the reception of the message disable_chv(chv1_ref, used_chv1) which represents the DISABLE CHV function. This transition is also conditioned by the logical expression used_chvl = chvl, which represents the fact that the chv used by DISABLE CHV, is equal to the chv of the card. The expected status for this reception is 90 00. Next transitions represent the sub-case 3 of the CHANGE CHV test.

The left one to $\mathrm{B}$ is conditioned by the reception of the message change_chv(chv1_ref, used_chv1, new_chv1), which represents the CHANVE CHV function. This transition is also conditioned by the logical expression used_chvl !=chv1 which represents the fact that the chv used by CHANGE CHV is not equal to the chv of the card. The expected status for this reception is 9804 . C 


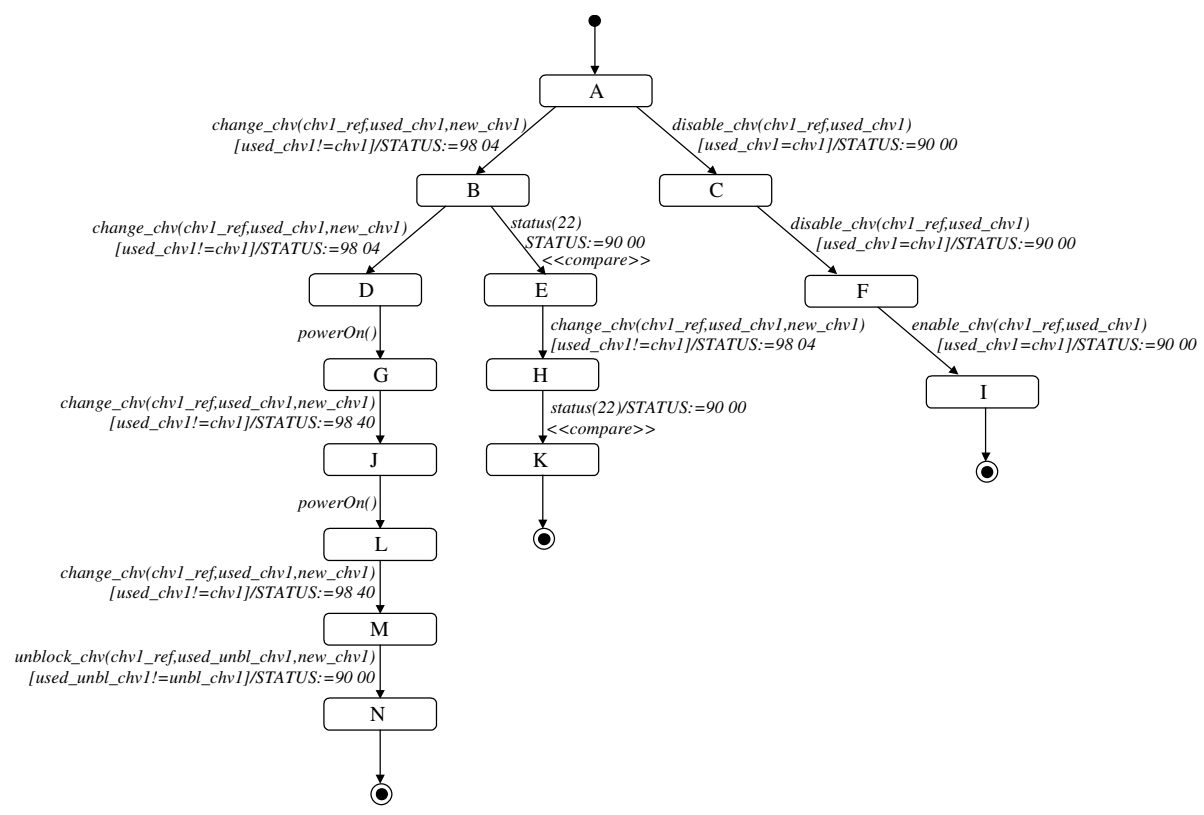

Fig. 4. The 3GPP 11.17 test of the CHANGE CHV function

holds two output transitions. The right path represents the sub-case 1 of the CHANGE CHV test and the left one the sub-case 2.

A status verification is represented by a transition conditioned by the reception of message $\operatorname{status}(n)$ where $n$ represents the size of the data to verify, in byte, 22 for our example. The expected status for this reception is 9000 . The data to compare are given in the $<<$ compare $>>$ stereotype. For example, on the transition from $\mathrm{B}$ to E, the <<compare $>>$ stereotype contains: $x x x x \quad x x x x \quad x x x x \quad x x \quad x x x x x x x \quad x x \quad x x \quad x x \quad x x \quad x x$ $x x \quad x x \quad x x \quad 83 \quad x x x x x x$ which means the $19^{\text {th }}$ byte is 83 and other bytes are any value, denoted $\mathrm{x}$.

\subsection{A UML Representation for Smart Card Specification}

With a UML state diagram, we can also represent a function specification. This diagram contains all the behaviours of a function in the same way as for a test. This abstract vision allows representing a function specification exhaustively. For example, on the CHANGE CHV function, this diagram has to represent a case with a direct correct CHANGE CHV, which is not considered in Figure 4.

The CHANGE CHV Function Example: UML Representation for Specification. To represent the CHANGE CHV specification exhaustively, we consider the $3 \mathrm{GPP}$ 11.11 standard given before. The corresponding state diagram is presented in Figure 5. 


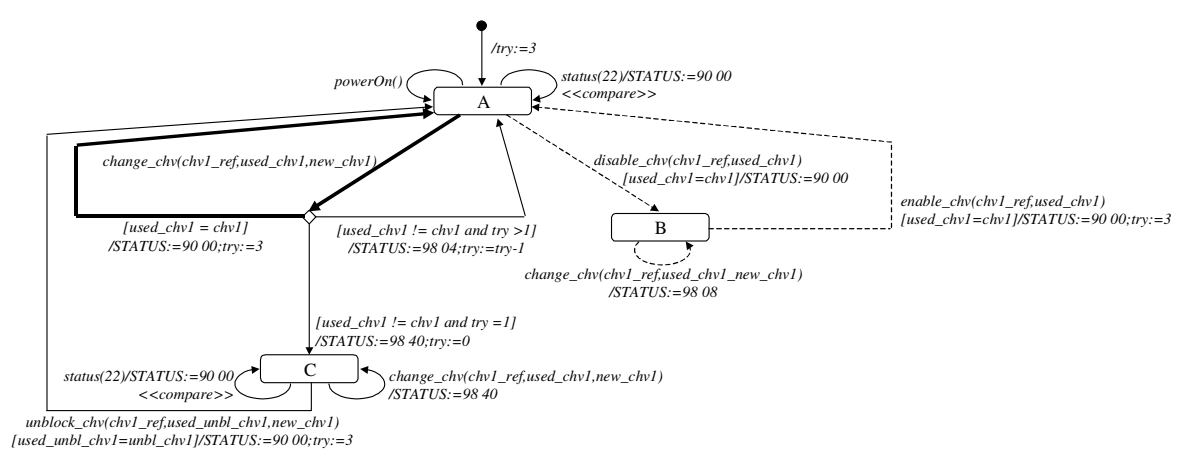

Fig. 5. Example of an abstract state-transition diagram for the CHANGE CHV functionalities

As we abstract the behaviour of the function, we introduce a counter try, which represents the number of attempts to change a chv. The initial state is $\bullet$. The output transition of the initial state is improved with the initialization of the try variable to 3 as mentioned in the specification.

The sub-case 1 of the specification is represented with bold lines: a change_chv(chvl_ref, used_chvl, new_chvl) message is received. The used_chvl is equal to $c h v 1$ so the expected status is 9000 , the $c h v l$ is changed to new_chvl and we can verify the status by the transition form A to A with the status message. As this diagram is an abstraction of the specification, the $<<$ compare $>>$ stereotype contains:

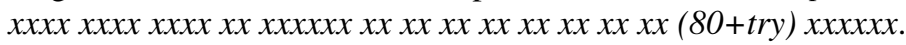

The sub-case 2 of the specification is represented with normal lines: a change_chv is received. The used_chvl is not equal to $c h v 1$ so the expected status is 9404 if try is different from 1 and is 9840 if try is equal to 1 . If status is 9404 , the transition goes in $A$ and we can verify the status or reset the session card, which has no impact on the variable try and so on the remaining number of attempts to change. If the status is 98 40 , the card is blocked and the transition goes in $\mathrm{C}$ where we can verify the status and receive change_chv messages. As the card is blocked, nothing appends. Except if an unblock_chv(chv1_ref, used_unblock_chv1, new_chv1) message is received with used_unblock_chvl equal to unblock_chv1.

We improved the specification with a behaviour described in the 3GPP 11.17 test but missing in the 3GPP 11.11 specification: the use of a correct DISABLE CHV before a CHANGE CHV. This behaviour is designed with dashed lines.

\subsection{Automatic Test Generation for UML Smart Card Model}

In part 2.2, we present how the AGATHA tool can generate automatically test cases. We applied this tool to our UML diagrams.

Firstly, as our representation of smart card test is very sequential, we use a coverage of transitions to compute a set of symbolic test cases. In that case in our example, AGATHA computes three paths. AGATHA extracts three numerical test cases. For a card that validates the $3 \mathrm{GPP} 11.17$ tests, it also validates these automatically generated tests. 
Secondly, for our representation of smart card specification, we use a more complicated criterion, the inclusion one to cover all the symbolic behaviours. In that case in our example, AGATHA computes more than two hundred paths, each of them corresponding to a symbolic behaviour. On a card that validates the 3GPP 11.17 tests, it has also to validate these automatically generated tests. It could be impressive to pass two hundred tests for a simple function but we test all possible behaviours of the CHANGE CHV function. Current works on AGATHA will certainly permit to reduce this number of tests with some optimization associated to the inclusion criteria. But in our case this reduction will not be very important due to the fact that the number of distinct symbolic behaviours associated to our example remains very close to the present one calculated by AGATHA: this is the price of exhaustiveness.

\section{Related Work and Conclusion}

In this article, we have summarized a solution to automatically generate tests for smart card functions. Assuming the validity of our approach, we have presented an automatic test generator, AGATHA based on symbolic execution techniques. We have also presented a way to design smart card functions with UML state diagram. We have used AGATHA on our UML diagrams and exposed obtained results. This first experience shows that it is possible to generate tests for smart card functions in an automatic way. Surely, and this is our first objective, our approach has to be used in a real context and in a complete development cycle of a smart card to completely improve its efficiency. We could reasonably hope an increase of the coverage and quality of test for each function taken separately.

Our approach is closed to the one developed in STG [22]. However, in STG, the test purposes must be defined by an expert. In that case, we may obtain « clever» test purposes but we have no way to measure the specification coverage. On the contrary, AGATHA suggests a limited number of predefined test purposes linked to structural or semantical coverage criteria. In that case, the set of generated tests allows to control with a great confidence the level of specification coverage.

LTG, the LEIRIOS test generator [26], uses classical structural coverage criteria which limit the combinatory of generated test cases. AGATHA also proposes criteria based on the analysis of the specification behaviours. Such criteria may be more accurate when generating test cases but can also be more subject to combinatory explosion. To avoid this problem, we are currently introducing some heuristics which allows to reasonably limit the number of generated test cases.

The use of UML state diagrams to design smart card behaviours allows us to consider more global behaviours that mix different smart card functions. Then we test the card rigorously and monitor the results. We also could consider atypical (or negative) tests that allow verifying smart card reactions outside of the admissible input domain defined by the specification. In this context, we could ensure a complete validation of a smart card.

Last point, as AGATHA is not only a test generator, we consider validating smart card properties corresponding to a security policy as defined for example in common criteria. In this context, we could ensure security properties of smart cards. 


\section{Acknowledgements}

The authors would like to thanks Clément Simon for his precious help during the development of this project. They also would like to thanks the CARDIS'06 reviewers and David Montouroy for their proofreading and their constructive remarks.

\section{References}

[1] J.R. Abrial, The B-Book, Assigning programs to meanings, Cambridge University Press, 1996

[2] S. Behnia, H. Waeselynck, Test criteria definition for B models, in procs. of the World Congress on Formal Methods (FM'99), vol.1708 of LNCS, pp.509-529, Toulouse (France), 1999

[3] E. Bernard, B. Legeard, X. Luck, F. Peureux, Generation of test sequences from formal specifications: GSM 11.11 standard case-study, The Journal of Software Practice and Experience, vol.34.10 pp.915-948, 2004

[4] C. Bigot, A. Faivre, J.P. Gallois, A. Lapitre, D. Lugato, J.Y. Pierron, N. Rapin, Automatic test generation with AGATHA, TACAS, 7-11 April 2003

[5] F. Bouquet, F. Peureux, Generation of functional test sequences from B formal specifications - Presentation and industrial case-study, in procs. of the $16^{\text {th }}$ International Conference on Automated Software Engineering (ASE'01), pp.377-381, San Diego (USA), November 2003

[6] F. Bouquet, B. Legeard, F. Peureux, E. Torreborre, Mastering Test Generation from Smartcard Software Formal Models, in Procs of the International Workshop on Construction and Analysis of Safe, secure and Interoperable Smart devices (CASSIS'04), vol.3362 of LNCS, pp.70-85, Marseille (France), March 2004

[7] O. Carre, H. Martin, J.J. Vandewalle, A semi formal model of Java Card 2.1 in UML, in 1st Gemplus Developer Conference, Paris, France, June 21-22, 1999

[8] D. Clarke, T. Jéron, V. Rusu, E. Zinovieva, Automated test and oracle generation for smart-card applications, in procs. of the International Conference on Research in Smartcards (e-Smart'01), vol.2140 of LNCS, pp.58-70, Cannes (France), September 2001

[9] E.M. Clarke, O. Grumberg, D.A. Peled, Model Cheking, The MIT press, 1999

[10] L.A. Clarke, A System to Generate Test Data and Symbolically Execute Programs, IEEE Transactions on Software Engineering, vol.SE-4 n.3, PP.178-187, September 1976

[11] J. Dick, A. Faivre, Automating the generation and sequencing of test cases from modelbased specifications, in procs. of the International Conference on Formal Methods Europe (FME'93), vol.670 of LNCS, pp.268-284, April 1993

[12] European Telecommunications Standards Institute, F-06921 Sophia Antipolis (France), GSM 11.11 v7.2.0 Technical Specification, 1999

[13] European Telecommunications Standards Institute, F-06921 Sophia Antipolis (France), GSM 11.17 v8.1.0 Technical Specification, 1999

[14] A. Faivre, C. Gaston, Test generation methodology based on symbolic execution for the Common Criteria higher levels, in MoDeVa workshop, Montego Bay (Jamaica), October 2005

[15] J.C. Fernandez, C. Jard, T. Jéron, C. Viho, Using on the fly verification techniques for the generation of test suites, Proceedings of CAV'96, LNCS 1102, Springer, New Brunswick, n.46, pp.145-150, 1997

[16] J.P. Gallois, A. Lanusse, Le test structurel pour la vérification de spécifications de systèmes industriels, Génie logiciel n.46 pp.145-150, 1997 
[17] J.P. Gallois, A. Lapitre, P. Lé, Analyse de spécifications industrielles et génération automatique de tests, ICSSEA'99, CNAM-Paris, France, 8-10 décembre, 1999

[18] M. Hennessy, H. Lin, Symbolic bisimulations, Theorical Computer Science, Vol.138 pp.353-389, Elsevier, 1995

[19] R. Hierons, Testing from Z specification, The journal of Software Testing, Verification and Reliability, vol.7 pp.19-33, 1997

[20] International Union of Telecommunications, Langage de programmation - Langage de Description et de Spécification du CCITT - Norme 34, Recommandation UIT T Z.100, March 1993

[21] M. Ishisone, T. Sawada, Brute: brute force rewriting engine, GAIST, http://www.theta. theta.ro/ cafeobj, January 2001

[22] B. Jeannet, T. Jéron, V. Rusu, E. Zinovieva, Symbolic Test Selection Based on Approximate Analysis, pp. 349-364, TACAS'05, Edinburgh (UK), April 2005

[23] W. Kelly, V. Maslov, W. Pugh, E. Rosser, T. Shpeisman, D. Wonnacott, The Omega Library version 1.1.0, University of Maryland, http://www.cs.umd.edu/projects/omega, November 1996

[24] J.C .King, Symbolic Execution and Program Testing, communications de l'ACM, vol.19 n.7, pp.385-394, July 1976

[25] A. Lapitre, Procédure de réduction pour les systèmes à base d'automates communicants : formalisation et mise en oeuvre, Phd Thesis, University of Paris XI, in collaboration with the CEA, December 2002

[26] LEIRIOS tool, http://www.leirios.com/index.php

[27] H. Lin, Symbolic Transition Graph with Assignment, CONCUR'96, Springer-Verlag, LNCS, Pise (Italie), August 1996

[28] D. Lugato, C. Bigot, Y. Valot, Validation and automatic test generation on UML models: the AGATHA approach, STTT (Software Tools for Technology Transfer), vol.5 n.2 pp.124-139, March 2004, Springer

[29] H. Martin, L. du Bousquet, Automatic test generation for Java-Card applets, in Java card Workshop, Cannes (France), September 2000

[30] OMG, Unified Modelling Language 2.0, OMG, Rapport formel/2003-04-01, January 2003

[31] J. Philipps, A. Pretschner, O. Slotosch, E. Aiglestorfer, S. Kriebel, K.Scholl, Modelbased test case generation for smartcards, in procs. of the $8^{\text {th }}$ International Workshop on Formal Methods for Industrial Critical Systems (FMICS'03), vol.80 of ENTCS, Trondheim (Norway), June 2003

[32] J.Y. Pierron, J.-P. Gallois, E. Fievet, A. Lapitre, D. Lugato, Validation de systèmes industriels par le test symbolique sur spécification STATEMATE, ICSSEA'00, CNAMParis, France, December 5-8, 2000

[33] J.Y. Pierron, Définition de critères de sélection de tests fonctionnels pour la validation des systèmes électroniques embarqués, Phd Thesis, University of Evry, France, in collaboration with the CEA and PSA, April 2003

[34] N. Rapin, Validation de spécification à base d'automates par des techniques de dépliages et d'exécution symbolique, Phd Thesis, University of Evry (France), in collaboration with the CEA and Ligeron S.A., July 2004

[35] P. Wolper, P.Godefroid, Partial-Order Methods for Temporal Verification, procs. of CONCNUR'93, pp.233-246, Hildesheim (Belgium), August 1993

[36] S. Yovine, Kronos: A verification tool for real time systems, Springer International Journal of Software Tools for Technology Transfer, vol. 1 n.1/2, October 1997 